\title{
Impact of Stimulus Polarity on oVEMP Response Using the Belly-Tendon Electrode Montage
}

DOI: $10.3766 /$ jaaa.17089

\author{
Daniel J. Romero* \\ Blythe H. Kitner* \\ Matthew J. Wilson* \\ Kaylee J. Smith $\dagger$
}

\begin{abstract}
Background: One stimulus parameter not well established with respect to the ocular vestibular evoked myogenic potential (oVEMP) is stimulus polarity. Many research studies traditionally record oVEMPs using alternating polarity primarily.

Purpose: The purpose of this study was to evaluate the effects of stimulus polarity on the oVEMP response under three different conditions (condensation, rarefaction, and alternating) with updated but established recording procedures-the belly-tendon electrode montage.
\end{abstract}

Research Design: oVEMPs were assessed with changes in stimulus polarity in the seated upright position.

Study Sample: Thirty otologically normal participants (60 ears) with no history of hearing or balance disorders and normal middle ear function.

Data Collection and Analysis: Five hundred-hertz air-conducted tone bursts at 95-dB nHL were used to evoke the oVEMP response while recordings were made from the contralateral eye to acoustical stimulation using the belly-tendon electrode montage. Measurements were made using three polarities: alternating, condensation, and rarefaction. Natus Bio-logic AEP hardware and software was used for all data collection and analysis.

Results: Condensation stimulus phase provided the largest response amplitude compared with alternating and/or rarefaction. Rarefaction provided the earliest latency among stimulus polarities.

Conclusions: Condensation is a more effective stimulus polarity regarding response amplitude when recording the oVEMP. This study further supports the use of the belly-tendon electrode montage for recording the oVEMP response.

Key Words: electrode montage, latency, N10 amplitude, otolith, phase-locking, response amplitude, stimulus polarity, utricle, vestibular evoked myogenic potential

Abbreviations: ACS = air conducted sound; LSD = least significant difference; oVEMP $=$ ocular vestibular evoked myogenic potential; RM-ANOVA = repeated measures analysis of variance; $\mathrm{SD}=$ standard deviation; VEMP = ocular vestibular evoked myogenic potential

\section{INTRODUCTION}

\section{The Vestibular Evoked Myogenic Potential (VEMP)}

The VEMP is a short-latency, biphasic, myogenic response (Colebatch and Halmagyi, 1992; Colebatch et al,
1994; Curthoys and Halmagyi, 1995). The ocular VEMP (oVEMP) is a subcategory of the VEMP response. The oVEMP can be recorded following stimulation of the utricle using high-level air-conducted sound (ACS) or bone-conducted vibration during a contraction of the inferior oblique muscle (Colebatch and Halmagyi, 1992; Halmagyi and Curthoys, 2000; McCaslin et al, 2008;

*Northern Illinois University, DeKalb, IL; †Henry Ford Health System, Detroit, MI

Corresponding author: Matthew J. Wilson, School of Allied Health \& Communicative Disorders, NIU-Speech-Language-Hearing Clinic, Northern Illinois University, DeKalb, IL 60115; Email: mwilson@niu.edu

Presented in part as a research poster at the American Balance Society Meeting, Scottsdale, AZ, February 28, 2017. 
Todd et al, 2009; Curthoys et al, 2011). The oVEMP carries afferent (excitatory) signals through the superior vestibular nerve, vestibular nuclei, and medial longitudinal fasciculus and, finally, terminates at the motor neurons of the contralateral inferior oblique muscle of the eye (Todd et al, 2007). Numerous studies have confirmed that the presence of the oVEMP signifies the integrity of the utricle and superior vestibular nerve (Todd et al, 2008; 2009; Curthoys, 2010; Govender et al, 2011).

\section{VEMP Stimulus Parameters}

Since its first observation, various acquisition protocols have been studied in the context of the oVEMP response in an effort to identify how best to objectively measure response characteristics of the utricle (Rosengren et al, 2005; Chihara et al, 2007; Todd et al, 2007; Iwasaki et al, 2008; Todd et al, 2009; Curthoys, 2010; Curthoys et al, 2011; Murnane et al, 2011; Piker et al, 2011; Cheng et al, 2012; Sandhu et al, 2013: 568; Makowiec et al, 2017). As a result of this work, it is now well understood that the overall response amplitude is affected by the interaction between stimulus characteristics, such as frequency, type, and intensity, and physical recording parameters such as electrode placement.

For instance, Murnane et al (2011) investigated the effect of electrode placement (ipsilateral versus contralateral) and stimulus level using a $500-\mathrm{Hz}$ tone burst in thirty individuals. Using a traditional infraorbital montage and alternating polarity, the results suggested that a contralateral, $500-\mathrm{Hz}$ tone burst at $125-\mathrm{dB}$ SPL provided the largest response. A later study by Cheng et al (2012) examined the influence of stimulus type by comparing click versus tone burst stimuli with four different ramp and plateau times in a population of 22 healthy adults. Responses were collected using a traditional, infraorbital montage and rarefaction polarity. The authors found tone burst versus click stimuli generated larger N1-P1 response amplitude with a difference of $10.94 \mathrm{uV}$ compared with $3.4 \mathrm{uV}$, respectively. Cheng et al (2012) also reported a lack of difference in the N1-P1 amplitude between the four types of tone burst stimuli with different rise and fall times (9.7$10.94 \mathrm{uV})$. Based on this work and that of other researchers (for review, see Curthoys and Grant, 2015), it is well established that a contralateral, $500-\mathrm{Hz}$ tone burst elicited by air-conducted sound activates receptor cells in the utricular maculae and produces an oVEMP response (Todd et al, 2008; 2009).

In conjunction with changes in stimulus characteristics, recent studies have shown that electrode placement can also cause variation in the response amplitude because of reference contamination (Piker et al, 2011; Sandhu et al, 2013; Govender et al, 2016a). The studies by Sandhu et al (2013) and Govender et al (2016a) completed serial cross-comparisons among different montages of the oVEMP using standard stimulus parameters (500-Hz tone burst at $95-\mathrm{dB} \mathrm{nHL}$ ) with alternating polarity. The central theme of the two studies was to determine the best recording sites for the oVEMP, that is, ones that provide the largest response amplitude with the least amount of reference contamination. Both studies used electrodes that were placed in locations ranging from the lateral to the inner canthus of the eye; however, Govender et al (2016a) excluded a nason placement. Both studies reported that infraorbital electrode placement resulted in smaller amplitudes than those that are more mediolateral. Specifically, Sandhu et al (2013) found recording from the belly tendon and nason, that is, belly-tendon montage, provided the largest amplitude. Although the lack of a nason placement in Govender et al (2016a) makes comparing results from the two studies difficult, later studies have confirmed the validity of using the belly-tendon montage to maximize the oVEMP response amplitude (Leyssens et al, 2017; Makowiec et al, 2017).

In all aforementioned studies, no attempt was made to look specifically at the influence of polarity, coupled with the belly-tendon electrode montage, on the overall response amplitude. One recent study by Amorim et al (2017) focused on the effects of polarity, but the authors used an electrode montage that resembled Govender et al (2016b) and not Sandhu et al (2013). Amorim and colleagues tested 12 healthy individuals using rarefaction, condensation, and alternating polarities. Both rarefaction and condensation produced waveforms that contained up to three different subpeaks. Results indicated amplitude values differed depending on which subpeak was examined. When focusing on only the most prominent subpeak, there was no significant difference between the three polarities; however, if comparing only the first main subpeak, the amplitudes were significantly larger for condensation (Amorim et al 2017).

In reviewing the literature, there are a paucity of studies that have examined how the response amplitude is affected by stimulus polarity (condensation, rarefaction, and alternating) using the belly-tendon montage specifically. Therefore, the purpose of the present investigation was to explore the effect of three stimulus polarities: rarefaction, condensation, and alternating, while using the belly-tendon montage on the oVEMP response. It was hypothesized that a larger response amplitude would be observed using a single polarity, specifically condensation, relative to rarefaction or alternating.

\section{METHODS}

A pproval for all data collection procedures was $A$ obtained from the university's Institutional Review Board before undertaking data collection. Thirty 
participants, recruited from the local university and surrounding community, took part in the study. Participants ranged from 19 to 32 years of age $(M=24.28$ years, standard deviation [SD] $=3.05$ years), and all were assessed using the same electrode montage to allow for cross-comparison. Before testing, informed consent was obtained from all participants. A series of questions were asked regarding aspects of hearing, balance, and overall health, including symptoms of ear pain, fullness, tinnitus, hearing loss, and dizziness/ imbalance for screening purposes. Tympanometry and acoustic reflexes were performed to verify normal middle ear function and used to contraindicate participation in the study - as an abnormal middle ear status may produce inaccurate responses when recording the oVEMP (Bath et al, 1999). Conventional audiometry was completed to ensure participants had normal hearing sensitivity from 250 to $8000 \mathrm{~Hz}$ using ER-3A insert earphones. Speech audiometry was presented at a comfortable loudness level and was used to further verify hearing sensitivity and speech understanding. Participants were excluded if they met any of the following criteria: abnormal hearing sensitivity between 250 to 8000 $\mathrm{Hz}$, abnormal middle ear status, history of balance disorders based on case history and/or questionnaires, history of neurological disorders, history of eye surgery (they were not excluded if they wore corrective contact lenses), history of drug or alcohol dependency, and history of neck injury or neck surgery.

\section{Procedure}

Participants were tested individually in the University's vestibular laboratory. Following the hearing evaluation, oVEMP responses were recorded while participants sat in an upright position and directed their gaze $30^{\circ}$ upward. Disposable, multipurpose snap electrodes were placed in five different positions around the face. Two noninverting electrodes were placed on the area around the belly tendon of the inferior oblique muscle, whereas two inverting electrodes were placed at the inner canthus of the eye with the ground electrode on the forehead (Figure 1).

During data collection, a 500-Hz tone burst at $95-\mathrm{dB}$ $\mathrm{nHL}$ was presented through ER3 inserts at a rate of 5.1 stimuli/second. Individual waveforms comprised one hundred fifty sweeps and were collected twice to judge replicability. As such, each participant's dataset consisted of 12 total waveforms ( 2 waveforms $\times 3$ conditions $\times 2$ ears). Further recording parameters and testing conditions can be found in Table 1. All data were collected with Natus Bio-logic hardware with clinical VEMP AEP software (Bio-logic Systems Corp., Mundelein, IL). Collection of data was randomized across polarities to avoid fatigue and potential collection effects.

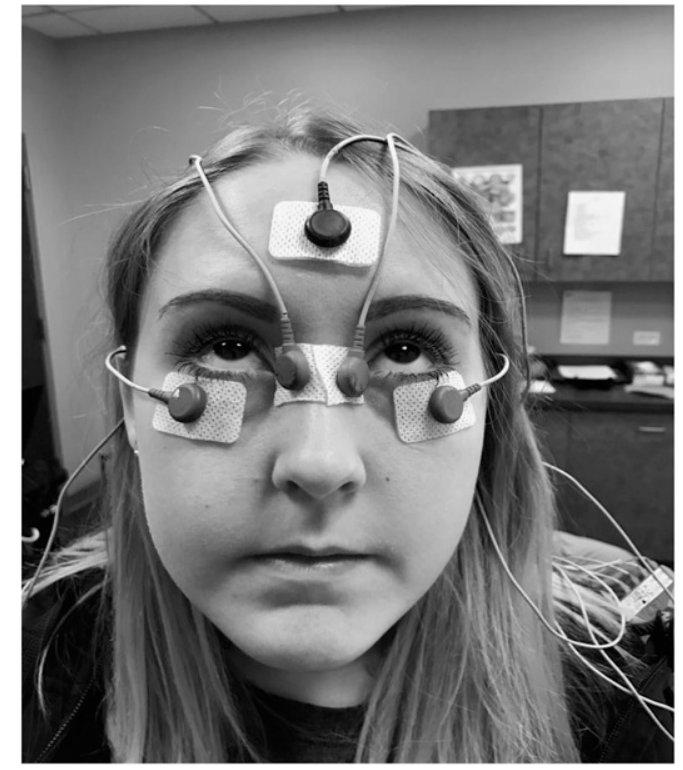

Figure 1. Electrode montage from Sandhu et al (2013) - the belly-tendon electrode montage. Active electrodes are placed on the belly tendon of the inferior oblique muscle and reference electrode at the inner canthus position.

\section{Data Analysis}

Before data analysis, individual oVEMP waveforms were grand averaged. Waveforms were then analyzed for the N10 and P15 latency, N10 absolute amplitude, interlatency, and interamplitude. Repeated measures analysis of variance (RM-ANOVA) was used to examine differences in these measures. For all RM-ANOVAs, the factor was stimulus polarity (repeated on three levels: alternating, condensation, and rarefaction). During the initial phase of data analysis, ear (repeated on two levels: left and right) was also included as a factor; however, ear was determined to be a nonsignificant factor in all comparisons. As such, the results reported below are collapsed across ears. Greenhouse-Geisser correction factors were used in instances where the sphericity assumption was violated. Epsilon values and corrected $p$ values are presented, but original degrees of freedom are maintained.

\section{RESULTS}

$\mathrm{R}$ eplicable responses were obtained from all 30 participants for each polarity in each ear. Means and SDs for component amplitude, latency, interamplitude, and interlatency by polarity are presented in Table 2 .

RM-ANOVA testing on N10 and P15 latency revealed a significant main effect for polarity $\left[F_{(2,58)}=42.376, p\right.$ $<0.001$, and $\left.\eta^{2}=0.594\right]$. None of the other main effects or interactions were significant. Follow-up least significant difference (LSD) testing on the polarity main effect for N10 indicated that condensation was longer 
Table 1. Recording Parameters and Testing Conditions

\begin{tabular}{ll}
\hline Parameters & \multicolumn{1}{c}{ Selection } \\
\hline Stimulus parameters & Insert earphones \\
Transducer & $500-\mathrm{Hz}$ tone burst \\
Stimulus & $0.50 / 2.00 / 0.50$ \\
Rise/plateau/fall (cycles) & Alternating, condensation, \\
Polarities & and rarefaction \\
& $95-\mathrm{dB} \mathrm{nHL}$ \\
Stimulus intensity & $5.1 \mathrm{stimuli} / \mathrm{second}$ \\
Presentation rate & 150 \\
Total number of trials & \\
Acquisition parameters & $\mathrm{Head}$ upright \\
Head position & Upward/midline \\
Gaze & $\leq 5 \mathrm{kOhms}$ for all channels \\
Impedance & $3-1000 \mathrm{~Hz}$ \\
Bandpass filter setting & $100,000 \times$ \\
Gain & $32 \mathrm{msec}$ \\
Time window & $512 \mathrm{~Hz}$ \\
Sampling rate &
\end{tabular}

than either alternating ( $p=0.029)$ or rarefaction $(p<$ 0.001 ). Furthermore, rarefaction produced a longer latency than alternating (Table 2).

\section{Interamplitude between Stimulus Polarity}

Interamplitude was calculated by taking the difference between N10 and P15 absolute amplitudes, that is, N10-P15. RM-ANOVA testing indicated a significant main effect for polarity $\left[F_{(2,58)}=26.442, p<0.001, \eta^{2}=\right.$ 0.477]. Follow-up LSD testing suggested that condensation showed the largest response amplitude relative to alternating $(p<0.001)$ and rarefaction $(p<0.001)$, whereas alternating and rarefaction did not differ from one another $(p=0.379)$ (Table 2$)$.

\section{Interpeak Latency between Stimulus Polarity}

Interpeak latency was calculated by taking the difference between $\mathrm{N} 10$ and $\mathrm{P} 15$ absolute latencies, that is, P15-N10. RM-ANOVA testing indicated a significant main effect for polarity $\left[F_{(2,58)}=56.56, p<0.001\right.$, and $\left.\eta^{2}=0.661\right]$. Follow-up LSD testing suggested that rarefaction showed the longest interpeak latency relative to alternating $(p<0.001)$ and condensation $(p<0.001)$, whereas alternating and condensation did not differ from one another $(p=0.125)$.

\section{DISCUSSION}

$\mathrm{T}$ he central purpose of the present investigation was to evaluate the effect of stimulus polarity under three different stimulus phases using the belly-tendon electrode montage. We hypothesized that pairing a single-polarity tone burst, specifically condensation, with the belly-tendon electrode montage would produce larger oVEMP amplitudes relative to alternating or rarefaction polarities.

In examining the main effects of this study, we found that the N10 amplitude was largest under condensation compared with either alternating or rarefaction, confirming our hypothesis. There are limited studies which have looked at the effects of stimulus polarity on the N10 amplitude. The first study by Govender et al (2016b), which was a reanalysis of the study by Lim et al (2013), found that rarefaction and condensation showed no difference in amplitude. The second, a study by Amorim et al (2017), focused on phase differences with measurements of amplitude and presence of subpeaks in 12 healthy adults with a similar montage to that by Govender et al (2016b). All significant findings in Amorim et al (2017) were dependent on which subpeak was measured, the first or the most prominent peak. Consequently, no amplitude differences were found with the most prominent peaks; however, the first main subpeak showed significantly larger amplitude for condensation than the other polarities.

In theory, a larger response amplitude should be elicited by a positive stimulus phase (condensation) due, in part, to the translation-sensitive activation patterns of the utricle, a finding that has been supported by past research. For example, McCue and Guinan (1994) reported that otolith afferents differentially phase-lock depending on the polarity of an incoming stimulus, suggesting that positive and negative pressure can influence activation patterns. A more recent review article by Curthoys and Grant (2015) described strong evidence of utricular and saccular phase-locking ability

Table 2. Means and SDs for the N10 and P15 Latency, Amplitude, Interamplitude, and Interpeak Latency by Polarity

\begin{tabular}{|c|c|c|c|c|}
\hline \multirow[b]{2}{*}{ Component } & \multirow[b]{2}{*}{ Measure } & \multicolumn{3}{|c|}{ Polarity } \\
\hline & & Rarefaction & Alternating & Condensation \\
\hline \multirow[t]{2}{*}{$\overline{N 10}$} & Amplitude & $11.84(7.69)$ & $11.57(6.95)$ & $12.59(6.67)$ \\
\hline & Latency & $9.17(0.60)$ & $9.59(0.53)$ & $9.74(0.50)$ \\
\hline P15 & Latency & $15.07(1.08)$ & $14.67(1.11)$ & $14.92(1.07)$ \\
\hline N10-P15 & Interamplitude & $21.30(11.86)$ & 20.65 (9.98) & $25.44(13.28)$ \\
\hline N10-P15 & Interpeak latency & $5.91(0.76)$ & $5.11(0.86)$ & $5.23(0.78)$ \\
\hline
\end{tabular}

Notes: Values are given in microvolts (SD) for amplitude and interamplitude; latency is given in milliseconds (SD) for latency and interpeak latency. 

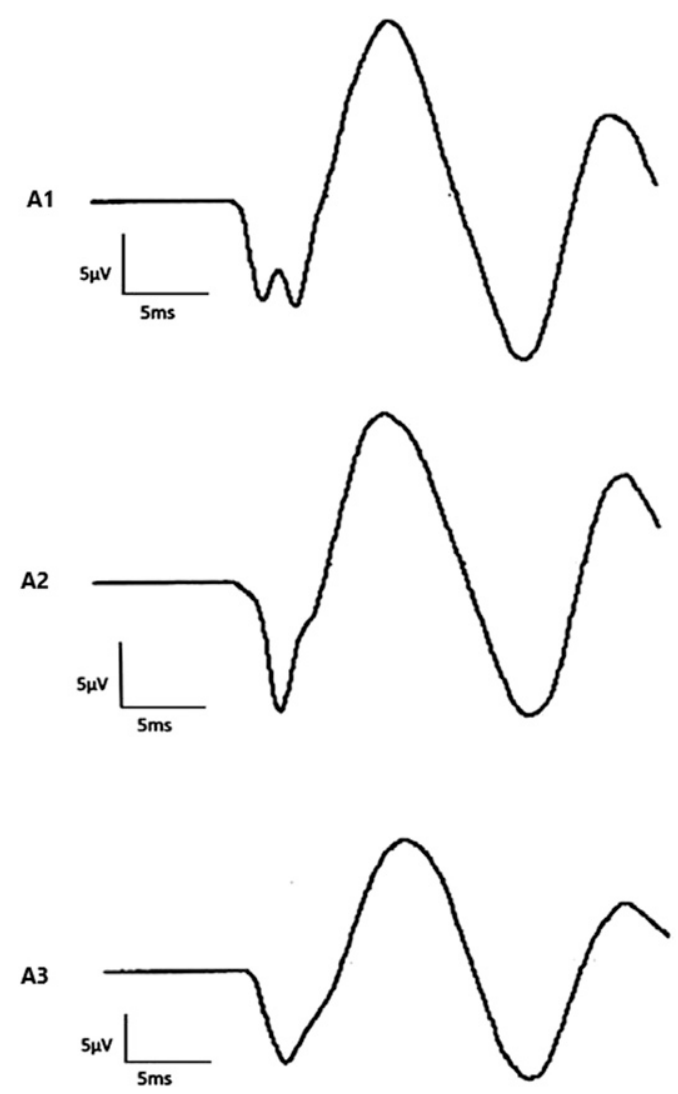

Figure 2. Grand-averaged waveforms from one participant representing morphological differences between three different stimulus polarities. $\mathrm{A} 1=$ rarefaction, $\mathrm{A} 2=$ condensation, and $\mathrm{A} 3=$ alternating.

to an incoming stimulus up to $2000 \mathrm{~Hz}$ for an air-conducted signal. They suggest that otoconia activation is heavily dependent on the incoming stimulus frequency. Specifically, low-frequency stimuli trigger mechanical deformation of the hair cells from the otoconial gel layer, whereas higher frequency stimuli caused activation via fluid pressure waves.

While analyzing the waveforms collected during the present study for amplitude, a secondary finding showed the N10 latency occurs earlier with rarefaction polarity than with condensation or alternating polarity (Figure 2).

The explanation of the latency difference found between condensation and rarefaction is supported by the anatomical arrangement of the stereocilia on either side of the striola, which activate hyperpolarization and depolarization simultaneously in the otoliths (McCue and Guinan, 1994; Leigh and Zee, 2006; Amorim et al, 2017). The medial portion of the macula in the utricle is activated by gravitational forces causing a pull action, and the lateral portion responds best to translational effects (push). The combination of the anatomical hair cell location on either side of the striola along with the push-pull activation of the hair cells may cause the latency differences found in this study and others (Lempert et al, 1998; Xue and Peterson, 2006; Curthoys, 2017; Curthoys et al, 2017). In addition, the type I and type II hair cell differences and location within the macula cause changes in phase as seen in a study by McCue and Guinan (1994). They proposed that afferent fibers from the saccule are classified into two types of short latency responses: push coming from activation by condensation and pull fibers coming from rarefaction activation when using a low-frequency tone burst greater than $80-\mathrm{dB}$ SPL. The movement from a positive to negative pressure wave causes the depolarization of type I hair cells as opposed to the onset of hyperpolarization within the mid-lateral region, an effect not observed with rarefaction or alternating stimulus phase.

\section{Limitations}

A possible limitation of the study was the control for gaze. Participants were asked to stare upward at midline $30^{\circ}$ at an earth-fixed target throughout the duration of the stimulus. It has been well established that the amplitude of the oVEMP is modulated based on the degree of gaze (Murnane et al, 2011). In addition, the electromyography of the participants' gaze was not monitored when obtaining the oVEMP recordings in our study. Weber et al (2012) also demonstrated active participation of the inferior rectus muscle during oVEMP recordings. Therefore, the role of other extraocular muscles including the inferior rectus muscle is not well understood in conjunction with the present stimulus and recording parameters.

\section{CONCLUSION}

$\mathrm{B}$ ased on data from the present study, condensation is a more effective stimulus polarity when recording and examining the oVEMP response amplitude using the belly-tendon electrode montage. The findings further support the use of the belly-tendon configuration proposed by Sandhu et al (2013). The oVEMP response is sensitive to the incoming stimulus phase, which may provide further clarification for the neuronal activity of the utricle because of its phasic and transient-driven response properties specific to each hair cell type. Future research should validate the effects found in this study and a reexamination of the age-dependent effects, asymmetry ratios, and pathological effects of otologic peripheral disorders to examine the contribution of the middle ear transfer function in phase-locking.

\section{REFERENCES}

Amorim FEAC, Sahdo AM, Giuliano LMP, Pinheiro DS, de Oliveira Braga NI, Manzano GM. (2017) Effects of the stimulus phase on the air-conducted ocular vestibular evoked myogenic potential in healthy subjects. Clin Neurophysiol 128:262-269. 
Bath A, Harris N, McEwan J, Yardley M. (1999) Effect of conductive hearing loss on the vestibulo-collic reflex. Clin Otolaryngol 24:181-183.

Cheng Y-L, Wu H-J, Lee G-S. (2012) Effects of plateau time and ramp time on ocular vestibular evoked myogenic potentials. $J$ Vestib Res 22:33-39.

Chihara Y, Iwasaki S, Ushio M, Murofushi T. (2007) Vestibularevoked extraocular potentials by air-conducted sound: another clinical test for vestibular function. Clin Neurophysiol 118: $2745-2751$.

Colebatch J, Halmagyi G. (1992) Vestibular evoked potentials in human neck muscles before and after unilateral vestibular deafferentation. Neurology 42:1635.

Colebatch J, Halmagyi G, Skuse N. (1994) Myogenic potentials generated by a click-evoked vestibulocollic reflex. J Neurol Neurosurg Psychiatry 57:190-197.

Curthoys IS. (2010) A critical review of the neurophysiological evidence underlying clinical vestibular testing using sound, vibration and galvanic stimuli. Clin Neurophysiol 121:132-144.

Curthoys IS. (2017) The new vestibular stimuli: sound and vibrationanatomical, physiological and clinical evidence. Exp Brain Res 235(4): 957-972.

Curthoys I, Grant J. (2015) How does high-frequency sound or vibration activate vestibular receptors? Exp Brain Res 233:691-699.

Curthoys IS, Halmagyi GM. (1995) Vestibular compensation: A review of the oculomotor, neural, and clinical consequences of unilateral vestibular loss. J Vestib Res 5(2):67-107.

Curthoys IS, Iwasaki S, Chihara Y, Ushio M, McGarvie LA, Burgess AM. (2011) The ocular vestibular-evoked myogenic potential to air-conducted sound; probable superior vestibular nerve origin. Clin Neurophysiol 122:611-616.

Curthoys IS, MacDougall HG, Vidal P-P, De Waele C. (2017) Sustained and transient vestibular systems: a physiological basis for interpreting vestibular function. Front Neurol 8:117.

Govender S, Cheng PY, Dennis DL, Colebatch JG. (2016a) Electrode montage and gaze effects on ocular vestibular evoked myogenic potentials (oVEMPs). Clin Neurophysiol 127:2846-2854.

Govender S, Rosengren SM, Colebatch JG. (2011) Vestibular neuritis has selective effects on air-and bone-conducted cervical and ocular vestibular evoked myogenic potentials. Clin Neurophysiol 122:1246-1255

Govender S, Rosengren SM, Dennis DL, Lim LJ, Colebatch JG. (2016b) Contrasting phase effects on vestibular evoked myogenic potentials (VEMPs) produced by air-and bone-conducted stimuli. Exp Brain Res 234:141-149.

Halmagyi G, Curthoys I. (2000) Otolith function tests. In: Herdman SJ, ed. Vestibular Rehabilitation. Philadelphia, PA: F.A. Davis, 196-214.

Iwasaki S, Smulders Y, Burgess A, McGarvie L, Macdougall H, Halmagyi G, Curthoys I. (2008) Ocular vestibular evoked myogenic potentials to bone conducted vibration of the midline forehead at Fz in healthy subjects. Clin Neurophysiol 119:2135-2147.
Leigh R, Zee D. (2006) The Neurology of Eye Movements, Edition 4 (Contemporary Neurology Series), New York, NY: Oxford University Press.

Lempert T, Gianna C, Brookes G, Bronstein A, Gresty M. (1998) Horizontal otolith-ocular responses in humans after unilateral vestibular deafferentation. Exp Brain Res 118(4):533-540.

Leyssens L, Heinze B, Vinck B, Van Ombergen A, Vanspauwen R, Wuyts FL, Maes LK. (2017) 'Standard' versus 'nose reference' electrode placement for measuring oVEMPs with air-conducted sound: test-retest reliability and preliminary patient results. Clin Neurophysiol 128:312-322.

Lim LJ, Dennis DL, Govender S, Colebatch JG. (2013) Differential effects of duration for ocular and cervical vestibular evoked myogenic potentials evoked by air- and bone-conducted stimuli. Exp Brain Res 224:437-445.

Makowiec K, McCaslin DL, Jacobson GP, Hatton K, Lee J. (2017) Effect of electrode montage and head position on air-conducted ocular vestibular evoked myogenic potential. Am J Audiol 26(2): $180-188$.

McCaslin DL, Jacobson GP, Harry T. (2008) The recordability of two sonomotor responses in young normal subjects. J Am Acad Audiol 19:542-547.

McCue M, Guinan J. (1994) Acoustically responsive fibers in the vestibular nerve of the cat. J Neurosci 14:6058-6070.

Murnane OD, Akin FW, Kelly JK, Byrd S. (2011) Effects of stimulus and recording parameters on the air conduction ocular vestibular evoked myogenic potential. J Am Acad Audiol 22:469-480.

Piker EG, Jacobson GP, McCaslin DL, Hood LJ. (2011) Normal characteristics of the ocular vestibular evoked myogenic potential. J Am Acad Audiol 22:222-230.

Rosengren S, Todd NM, Colebatch J. (2005) Vestibular-evoked extraocular potentials produced by stimulation with bone-conducted sound. Clin Neurophysiol 116:1938-1948.

Sandhu JS, George SR, Rea PA. (2013) The effect of electrode positioning on the ocular vestibular evoked myogenic potential to air-conducted sound. Clin Neurophysiol 124:1232-1236.

Todd NP, Rosengren SM, Colebatch JG. (2009) A utricular origin of frequency tuning to low-frequency vibration in the human vestibular system? Neurosci Lett 451:175-180.

Todd NPM, Rosengren SM, Aw ST, Colebatch JG. (2007) Ocular vestibular evoked myogenic potentials (OVEMPs) produced by air-and bone-conducted sound. Clin Neurophysiol 118:381-390.

Todd NPM, Rosengren SM, Colebatch JG. (2008) A source analysis of short-latency vestibular evoked potentials produced by air-and bone-conducted sound. Clin Neurophysiol 119:1881-1894.

Weber KP, Rosengren SM, Michels R, Sturm V, Straumann D, Landau K. (2012) Single motor unit activity in human extraocular muscles during the vestibulo-ocular reflex. J Physiol 590: 3091-3101.

Xue J, Peterson EH. (2006) Hair bundle heights in the utricle: differences between macular locations and hair cell types. $J$ Neurophysiol 95:171-186. 